\title{
Linial's Dual Conjecture for Path-Spine Digraphs
}

\author{
Vinícius de Souza Carvalho ${ }^{1 *}$, Cândida Nunes da Silva ${ }^{1 \dagger}$, Orlando Lee ${ }^{2 \ddagger}$ \\ ${ }^{1}$ Departament of Computing - Federal University of São Carlos (DComp-UFSCar) \\ Rod. João Leme dos Santos km 110 - SP-264, CEP 18052-780, Sorocaba - SP - Brazil \\ ${ }^{2}$ Institute of Computing - University of Campinas (IC-UNICAMP) \\ Av. Albert Einstein, 1251, Cidade Universitária, CEP 13083-852, Campinas - SP - Brazil \\ vc18051997@gmail.com, candida@ufscar.br, lee@ic.unicamp.br
}

\begin{abstract}
Given a digraph $D$, a coloring $\mathcal{C}$ of $D$ is a partition of $V(D)$ into stable sets. The $k$-norm of $\mathcal{C}$ is defined as $\sum_{C \in \mathcal{C}} \min \{|C|, k\}$. A coloring of $D$ with minimum $k$-norm has its $k$-norm noted by $\chi_{k}(D)$. $A$ (path)- $k$-pack of $a$ digraph $D$ is a set of $k$ vertex-disjoint (directed) paths of $D$. The weight of a $k$-pack is the number of vertices covered by the $k$-pack. We denote by $\lambda_{k}(D)$ the weight of a maximum $k$-pack. Linial conjectured that $\chi_{k}(D) \leq \lambda_{k}(D)$ for every digraph. Such conjecture remains open, but has been proved for some classes of digraphs. We prove the conjecture for path-spine digraphs, defined as follows. A digraph $D$ is path-spine if there exists a partition $\{X, Y\}$ of $V(D)$ such that $D[X]$ has a Hamilton path and every arc in $D[Y]$ belongs to a single path $Q$.
\end{abstract}

\section{Introduction}

In this paper we consider directed graphs without loops or parallel edges. For a digraph $D$, we denote by $V(D)$ its set of vertices and by $A(D)$ its set of arcs. Given an arc $a=(u, v) \in A(D)$, we say that $u$ and $v$ are adjacent and also that $u$ dominates $v$ and $v$ is dominated by $u$.

A (directed) path of a digraph $D$ is a sequence $P=\left(v_{1}, v_{2}, \ldots, v_{\ell}\right)$ of distinct vertices such that arc $\left(v_{i}, v_{i+1}\right) \in A(D)$, for every $1 \leq i<\ell$. We denote by $V(P)$ the set of vertices of $P$ and abuse notation denoting by $|P|$ the cardinality of $V(P)$. We denote by $\lambda(D)$ the maximum order of a path in $D$. Given a positive integer $k$, a collection of $k$ vertex-disjoint paths $\mathcal{P}^{k}=\left\{P_{1}, P_{2}, \ldots, P_{k}\right\}$ is called a (path) $k$-pack of $D$. A stable set of a digraph $D$ is a subset of pairwise non-adjacent vertices of $V(D)$. We denote by $\alpha(D)$ the cardinality of the maximum stable set in $D$. A coloring $\mathcal{C}$ of a digraph $D$ is a partition of $V(D)$ into stable sets; each stable set is a color class of $\mathcal{C}$. We denote by $|\mathcal{C}|$ the number of stable sets in the coloring. We say that a coloring $\mathcal{C}$ of $D$ is optimal if it has minimum cardinality (the least possible number of color classes) and we denote its cardinality by $\chi(D)$.

Gallai and Roy were the first ones to find a relationship between coloring and (path) $k$-packs. In two independent papers [Gallai 1968] and [Roy 1967] showed that $\chi(D) \leq \lambda(D)$ for every digraph $D$; this is known as Gallai-Roy's Theorem. Later,

\footnotetext{
*Support by CNPq (PIBIC-UFSCar program). ORCID: 0000-0002-6372-1898.

†ORCID: 0000-0002-4649-0274.

${ }^{\ddagger}$ Support by FAPESP (grant 2015/11937-9) and CNPq (grants 303766/2018-2 and 425340/2016-3). ORCID: 0000-0004-4462-
} 3325. 
in [Mirsky 1971] it was shown that $\chi(D)=\lambda(D)$ for every transitive acyclic digraph; this is known as Mirsky's Theorem.

Further attempts to generalize such results were made. New metrics of maximality and minimality were defined with respect to a positive integer $k$ for $k$-packs and for colorings. The weight of a $k$-pack $\mathcal{P}^{k}=\left\{P_{1}, P_{2}, \ldots, P_{k}\right\}$, denoted by $\left\|\mathcal{P}^{k}\right\|$, is given by $\sum_{P_{i} \in \mathcal{P}_{k}}\left|P_{i}\right|$. A $k$-pack is optimal if its weight is maximum and the weight of an optimal $k$-pack is denoted by $\lambda_{k}(D)$. The $k$-norm of a coloring $\mathcal{C}$ of $D$ is defined as $\sum_{C \in \mathcal{C}} \min \{|C|, k\}$ and denoted by $|\mathcal{C}|_{k}$. A coloring of $D$ with minimum $k$-norm is called $k$-optimal and we denote its $k$-norm by $\chi_{k}(D)$. Note that $\lambda(D)=\lambda_{1}(D)$ and $\chi(D)=\chi_{1}(D)$. In [Greene 1976], a generalization of Mirsky's Theorem was given by showing that $\chi_{k}(D)=\lambda_{k}(D)$ for every transitive acyclic digraph $D$ and every positive integer $k$; this is known as Greene's Theorem. Along the same line, Linial proposed a conjecture that sought to generalize Gallai-Roy's Theorem. It is stated below.

Conjecture 1 (Linial's Dual Conjecture [Linial 1981]). For every digraph $D$ and every positive integer $k$, we have $\chi_{k}(D) \leq \lambda_{k}(D)$.

Linial's Dual Conjecture is the main focus of this paper. This conjecture remains open, but has partial results. Recent contributions include [Sambinelli et al. 2017a, Sambinelli et al. 2019]. Other recent contributions on a related - but different - Conjecture of Linial include [Sambinelli et al. 2017b, Yoshimura et al. 2019], papers that present techniques that were important inspirations for this work. A thorough summary on the state of the art of both conjectures can be found in [Sambinelli 2018, Table 6.1]. In order to define the class of digraphs of interest in this paper, we need the following additional concepts. A Hamilton path is a path containing every vertex in $V(D)$. Digraph $D$ is traceable if it contains a Hamilton path. A tournament is a digraph $D$ for which every pair of vertices $u$ and $v$ are adjacent. We do know, by Rédei's Theorem (see [Bondy and Murty 2008]), that every tournament is traceable. We denote by $D[X, Y]$ a digraph with a partition $\{X, Y\}$ of $V(D)$. Depending on the structure of the subdigraphs $D[X]$ and $D[Y]$ induced by each part we may define several classes of digraphs. A digraph $D[X, Y]$ is split if $D[X]$ is a tournament and $D[Y]$ is a stable set. Consider a digraph $D[X, Y]$ such that $D[X]$ is traceable; then, $D$ is spine if $D[Y]$ is a stable set, arc-spine if $D[Y]$ contains at most one arc and path-spine if $D[Y]$ contains at most one nontrivial path $Q$ (if any). Clearly, path-spine digraphs are a superclass of arc-spine digraphs which, in turn, are a superclass of spine digraphs. Also, by Rédei's Theorem, spine digraphs are a superclass of split digraphs.

Here we prove Linial's Dual Conjecture for path-spine digraphs. One important partial result for this conjecture is due to Hartman, Saleh and Hershkowits, who proved in [Hartman et al. 1994] that it holds for split digraphs (such proof can be easily adapted for spine digraphs). Therefore, our result is, as far as we know, the first generalization of Hartman, Saleh and Hershkowits's proof for split digraphs since its publication in 1994.

\section{Path-spine digraphs and Linial's Dual Conjecture}

To prove the validity of Linial's Dual Conjecture we find an upper bound for $\chi_{k}(D)$ and a lower bound for $\lambda_{k}(D)$ aiming at minimizing the gap between these two values. To do so, we will define standard ways to obtain both a coloring and a $k$-pack for a digraph $D$. We will assume, without loss of generality, that the partition $\{X, Y\}$ considered makes $X$ 
maximal. Let $P$ be the Hamilton path in $D[X]$ and let $Q=\left(y_{1}, y_{2}, \ldots, y_{d}\right)$ be the single path in $D[Y]$ with $d$ vertices. A canonical k-pack for a path-spine digraph $D$ is defined as $\mathcal{P}^{k}=\{P\} \cup\{Q\} \cup\{(y): y \in S\}$, where $S$ is some arbitrary set of $k-2$ vertices in $Y^{\prime}=Y-V(Q)$. The weight of a canonical $k$-pack is therefore

$$
\left\|\mathcal{P}^{k}\right\|=|X|+\min \{|Y|-(d-2), k\}+(d-2) .
$$

Now, the definition of the standard coloring differ slightly depending on whether $d \geq 3$ or not. When $d \geq 3$, a canonical coloring for a path-spine digraph $D$ is defined as $\mathcal{C}=\left\{C_{1}\right\} \cup\left\{\{v\}: v \in V(D)-C_{1}\right\}$, where the unique non-trivial color class $C_{1}$ is defined as $C_{1}=Y^{\prime} \cup\left\{y_{1}, y_{d}\right\}$. The $k$-norm of such coloring is:

$$
|\mathcal{C}|_{k}=\min \left\{\left|Y^{\prime} \cup\left\{y_{1}, y_{d}\right\}\right|, k\right\}+|X|+(d-2) .
$$

Note that $\left|Y^{\prime} \cup\left\{y_{1}, y_{d}\right\}\right|=|Y|-(d-2)$, so $\| \mathcal{P}^{k}||$ and $|\mathcal{C}|_{k}$ coincide no matter what is the value of $\min \{|Y|-(d-2), k\}$. Therefore the conjecture trivially holds when $d \geq 3$. It thus suffices to prove the conjecture for the case $d=2$ since it is known to be valid for $d=1$. In such case, $D[Y]$ is a stable set and $D$ is in fact a spine digraph; Hartman, Saleh and Hershkowits' proof for split digraphs can be easily adapted to spine digraphs as mentioned before. We may thus assume that $d=2$, i. e., $D$ is an arc-spine digraph and the ends of $Q$ are adjacent. To simplify notation, we will denote $y_{1}$ by $u$ and $y_{2}=y_{d}$ by $v$ in this case. The definition of a canonical coloring for an arc-spine digraph differs from that of a path-spine solely on the non-trivial color class $C_{1}$, which is defined as $C_{1}=Y^{\prime} \cup\{u\}$. The $k$-norm of such coloring is thus:

$$
|\mathcal{C}|_{k}=\min \left\{\left|Y^{\prime} \cup\{u\}\right|, k\right\}+|X|+1 .
$$

When, $k \geq|Y|$, we have $|\mathcal{C}|_{k}=\| \mathcal{P}^{k}||=|X|+|Y|$ and the conjecture trivially holds. Therefore, we may henceforth assume that $k<|Y|$. Clearly, $|\mathcal{C}|_{k}-1=\left\|\mathcal{P}^{k}\right\|$; thus the gap between the bounds obtained for $\chi_{k}$ and $\lambda_{k}$ is 1 . As $Y-\{v\}$ is always a stable set, $\alpha(D) \geq|Y|-1$. When $\alpha(D)>|Y|-1$ we may define a coloring with a unique non-trivial color class composed by a maximum stable set. Such coloring would have $k$-norm smaller than $|\mathcal{C}|_{k}-1$, thus settling the conjecture. We may thus assume that $\alpha(D)=|Y|-1$ and it suffices to prove Theorem 1 to conclude the proof. The proof of this theorem relies on some lemmas, whose proofs are omitted due to space constraint. Before stating the lemmas, we need to define some rather technical concepts.

We say that a vertex $x \in X$ is $u v$-triangulated when $x$ is adjacent to both $u$ and $v$. We say that $D$ has an obstruction when for every $x \in X$ we have that: (a) $x$ is adjacent to some $y \in Y^{\prime}$; or (b) condition (a) is false and $x$ is $u v$-triangulated. A $t$-subset of an arc-spine digraph $D[X, Y]$ is a subset $T$ of $Y$ that has $t$ vertices and $D[X, T]$ has a path partition into $t-1$ paths. Let $P=\left(x_{1}, x_{2}, \ldots, x_{\ell}\right)$ be a Hamilton path of $D[X]$. We say that two vertices $x_{i}$ and $x_{j}$ in $X$ with $i<j$ in $P$ are the ends of a knot if the following three conditions are satisfied: (i) $x_{i}$ dominates some $y_{i}$ in $Y^{\prime}$; (ii) $x_{j}$ is dominated by some $y_{j}$ in $Y^{\prime}$; and (iii) $x_{i+1}, \ldots, x_{j-1}$ are $u v$-triangulated.

Lemma 1. If $D[X, Y]$ is an arc-spine digraph and $k=2$, then $\chi_{k}(D) \leq \lambda_{k}(D)$.

Lemma 2. Given two paths $P$ and $Q$ such that every vertex $p \in P$ is adjacent to every vertex $q \in Q$, then there is a path $R$ such that $V(R)=V(P) \cup V(Q)$.

Lemma 3. If $D[X, Y]$ is an arc-spine digraph such that $\alpha(D)=|Y|-1$, then $D$ has an obstruction. 
Lemma 4. If $D[X, Y]$ is an arc-spine digraph and $T \subset Y$ is a $t$-subset of $D$ such that $k \geq t-1$, then $\chi_{k}(D) \leq \lambda_{k}(D)$.

Lemma 5. Let $D[X, Y]$ be an arc-spine digraph and let $P=\left(x_{1}, x_{2}, \ldots, x_{\ell}\right)$ be a Hamilton path of $D[X]$. If there is a pair $x_{i}$ and $x_{j}$ that are ends of a knot, then $\left\{u, v, y_{i}, y_{j}\right\}$ is a 4-subset of $D$, where $y_{i}$ is some vertex in $Y^{\prime}$ dominated by $x_{i}$ and $y_{j}$ is some vertex in $Y^{\prime}$ that dominates $x_{j}$.

Theorem 1. If $D[X, Y]$ is an arc-spine digraph such that $\alpha(D)=|Y|-1$ and $k<|Y|$, then $\chi_{k}(D) \leq \lambda_{k}(D)$.

Proof. We assume that $k \geq 3$, by Lemma 1. By Lemma 3,D has an obstruction. Let $P=\left(x_{1}, x_{2}, \ldots, x_{\ell}\right)$ be a Hamilton path of $D[X]$. By Lemmas 4 and 5 if there are two vertices of $P$ that are ends of a knot in $D$, then $\chi_{k}(D) \leq \lambda_{k}(D)$. We thus assume this does not happen. If every vertex of $X$ is $u v$-triangulated, by Lemma 2, there is one path with all vertices in $X \cup\{u, v\}$, thus making $\{u, v\}$ a 2-subset. By Lemma 4 the conjecture holds in this case. So, some vertex $x_{i} \in X$ is not $u v$-triangulated and we assume $i$ is the smallest possible, without loss of generality. As $D$ has an obstruction, there is a vertex $y_{i} \in Y^{\prime}$ adjacent to $x_{i}$. If $y_{i}$ dominates $x_{i}$ then, by the maximality of $X$, we may assume $i>1$. Therefore, $\left\{u, v, y_{i}\right\}$ is a 3 -subset of $D$. Path $P_{1}=\left(y_{i}, x_{i}, x_{i+1}, \ldots, x_{\ell}\right)$ contains $y_{i}$ and Lemma 2 ensures the existence of a path $P_{2}$ containing the vertices in $\left(x_{1}, \ldots, x_{i-1}\right)$ as well as $u$ and $v$. Lemma 4 implies that the conjecture holds in this case. We may thus assume that $y_{i}$ is dominated by $x_{i}$. Let $j$ be the largest value so that $x_{j}$ is not $u v$-triangulated and $x_{j}$ dominates a vertex $y_{j} \in Y^{\prime}$. Clearly, $j \geq i$. We may assume $j<\ell$ by the maximality of $X$. By symmetry, we may also assume that some vertex of $\left(x_{j+1}, \ldots, x_{\ell}\right)$ is not $u v$-triangulated, otherwise $\left\{u, v, y_{j}\right\}$ would be a 3 -subset of $D$. Now let $h$ be the smallest value so that $j+1 \leq h \leq \ell$ and $x_{h}$ is not $u v$-triangulated. Since $D$ has an obstruction, there must be a vertex $y_{h} \in Y^{\prime}$ adjacent to $x_{h}$. By the choice of $j, x_{j}$ is dominated by $y_{h}$. But then, $x_{j}$ and $x_{h}$ are ends of a knot in $D$; a contradiction.

\section{References}

Bondy, J. A. and Murty, U. S. R. (2008). Graph Theory. Springer.

Gallai, T. (1968). On directed paths and circuits. Theory of graphs, pages 115-118.

Greene, C. (1976). Some partitions associated with a partially ordered set. Journal of Combinatorial Theory, Series A, 20(1):69-79.

Hartman, I. B.-A., Saleh, F., and Hershkowitz, D. (1994). On greene's theorem for digraphs. Journal of Graph Theory, 18(2):169-175.

Linial, N. (1981). Extending the Greene-Kleitman theorem to directed graphs. Journal of Combinatorial Theory, Series A, 30(3):331-334.

Mirsky, L. (1971). A dual of Dilworth's decomposition theorem. Amer. Math. Monthly, 78:876-877.

Roy, B. (1967). Nombre chromatique et plus longs chemins d'un graphe. ESAIM: Mathematical Modelling and Numerical Analysis-Modélisation Mathématique et Analyse Numérique, 1(5):129-132.

Sambinelli, M. (2018). Problemas de partição em grafos e digrafos. PhD thesis, Universidade Estadual de Campinas.

Sambinelli, M., da Silva], C. N., and Lee, O. (2017a). Advances in Aharoni-Hartman-Hoffman's conjecture for split digraphs. Electronic Notes in Discrete Mathematics, 62:111 - 116. Proceedings of LAGOS'17.

Sambinelli, M., da Silva, C. N., and Lee, O. (2017b). On Linial's conjecture for spine digraphs. Discrete Mathematics, 340(5):851-854.

Sambinelli, M., Negri Lintzmayer, C., Nunes Da Silva, C., and Lee, O. (2019). Berge's conjecture and AharoniHartman-Hoffman's conjecture for locally in-semicomplete digraphs. Graph. Comb., 35(4):921-931.

Yoshimura, L. R., Sambinelli, M., da Silva, C. N., and Lee, O. (2019). Linial's conjecture for arc-spine digraphs. Electronic Notes in Theoretical Computer Science, 346:735-746. 